\section{D) Check for updates}

Cite this: Dalton Trans., 2021, 50 1274

Received 25th September 2020 Accepted 21st December 2020 DOI: 10.1039/d0dt03339a rsc.li/dalton

\title{
The impact of boron atoms on clathrate-I silicides: composition range of the borosilicide $\mathrm{K}_{8-x} \mathrm{~B}_{y} \mathrm{Si}_{46-y}{ }^{\dagger}$
}

\author{
Walter Jung, ${ }^{\text {a,b }}$ Bodo Böhme, ${ }^{a}$ Julia M. Hübner, ${ }^{a}$ Ulrich Burkhardt, ${ }^{a}$ Horst Borrmann, ${ }^{a}$ \\ Matej Bobnar, ${ }^{a}$ Hong Duong Nguyen, ${ }^{a, c}$ Ingo Pantenburg, ${ }^{b}$ Martin Etter, ${ }^{d}$ \\ Ulrich Schwarz, (D) ${ }^{a}$ Yuri Grin ${ }^{a}$ and Michael Baitinger (D)*a
}

\begin{abstract}
The clathrate-I borosilicide $\mathrm{K}_{8-x} \mathrm{~B}_{y} \mathrm{Si}_{46-y}(0.8 \leq x \leq 1.2$ and $6.4 \leq y \leq 7.2$; space group $\mathrm{Pm} \overline{3} n)$ was prepared in sealed tantalum ampoules between $900{ }^{\circ} \mathrm{C}$ and $1000^{\circ} \mathrm{C}$. By high-pressure preparation at $8 \mathrm{GPa}$ and $1000{ }^{\circ} \mathrm{C}$, a higher boron content is achieved ( $x=0.2, y=7.8$ ). Crystal structure and composition were established from X-ray diffraction data, chemical analysis, WDX spectroscopy, and confirmed by ${ }^{11} \mathrm{~B}$ and ${ }^{29} \mathrm{Si}$ NMR, and magnetic susceptibility measurements. The compositions are electron-balanced according to the Zintl rule within one estimated standard deviation. The lattice parameter varies with composition from $a=9.905 \AA$ for $\mathrm{K}_{7.85(2)} \mathrm{B}_{7.8(1)} \mathrm{Si}_{38.2(1)}$ to $\mathrm{a}=9.968(1) \AA ̊$ for $\mathrm{K}_{6.80(2)} \mathrm{B}_{6.4(5)} \mathrm{Si}_{39.6(5)}$.
\end{abstract}

\section{Introduction}

Intermetallic clathrate-I phases comprise covalent frameworks of four-bonded atoms, which host electropositive metal atoms in 20-atom and 24-atom cages (Fig. 1). The framework topology matches the connectivity of group 14 atoms $4 b(\mathrm{E} 14)^{0}$ and group 13 Zintl-anions $4 b(\mathrm{E} 13)^{-}$, which form a variety of ternary representatives. $^{1-4}$ In particular, ternary silicon and germanium clathrates with $\mathrm{E} 13=\mathrm{Al},{ }^{5-11} \mathrm{Ga},{ }^{12-17} \operatorname{In}^{18-20}$ have been studied thoroughly, often motivated by the search for new thermoelectric materials. ${ }^{12,13}$ Clathrate compounds often exhibit a broad homogeneity range, and their compositions do not necessarily follow the Zintl rule. For example, in the ternary system $\mathrm{Ba}-\mathrm{Al}-\mathrm{Si}$, the homogeneity range covers compositions from $\mathrm{Ba}_{8} \mathrm{Al}_{6.9} \mathrm{Si}_{39.1}{ }^{10}$ to $\mathrm{Ba}_{8} \mathrm{Al}_{15} \mathrm{Si}_{31}{ }^{9}$ while the Zintl-rule composition $\mathrm{Ba}_{8} \mathrm{Al}_{16} \mathrm{Si}_{30}$ seems inaccessible. However, the rare clathrate-I representatives with boron atoms, $\mathrm{K}_{7} \mathrm{~B}_{7} \mathrm{Si}_{39}{ }^{21}$ and

${ }^{a}$ Max-Planck-Institut für Chemische Physik fester Stoffe, Nöthnitzer Straße 40, 01187 Dresden, Germany. E-mail: Michael.Baitinger@cpfs.mpg.de

${ }^{b}$ Department für Chemie, Institut für Anorganische Chemie, Universität zu Köln, Greinstraße 6, 50939 Köln, Germany

${ }^{c}$ Enviromental and Ressources Management Department, Quang Ninh Economic Zone Authority, Ha Long City, Vietnam

${ }^{d}$ Deutsches Elektronen-Synchrotron (DESY), 22607 Hamburg, Germany

$\dagger$ Electronic supplementary information (ESI) available: CSD numbers: Highpressure sample $\mathrm{K}_{7.85(2)} \mathrm{B}_{7.8(1)} \mathrm{Si}_{38.2(1)}$, CSD 2006316; crystal 1: $\mathrm{K}_{7.18(2)} \mathrm{B}_{7.2(5)} \mathrm{Si}_{38.8(5)}$, CSD 2046329; crystal 2: $\mathrm{K}_{7.12(4)} \mathrm{B}_{7.1(6)} \mathrm{Si}_{38.9(6)}$, CSD 2046333; crystal 3: $\mathrm{K}_{6.98(4)} \mathrm{B}_{6.4(5)} \mathrm{Si}_{39.6(5)}$, CSD 2046330; crystal 4, $\mathrm{K}_{7.08(1)} \mathrm{B}_{6.8(3)} \mathrm{Si}_{39.2(3)}$, CSD 2046332; crystal 5: $\mathrm{K}_{6.80(2)} \mathrm{B}_{6.4(5)} \mathrm{Si}_{39.6(5)}$, CSD 2046331. CCDC 2006316-2046333. For ESI and crystallographic data in CIF or other electronic format see DOI: 10.1039/ d0dt03339a
$\mathrm{Rb}_{8} \mathrm{~B}_{8} \mathrm{Si}_{38},{ }^{22}$ were found to follow the Zintl-Klemm concept. With four-bonded boron atoms, the electron balances are $\left[\mathrm{K}^{1+}\right]_{7}\left[\mathrm{~B}^{1-}\right]_{7}\left[\mathrm{Si}^{0}\right]_{39}$, and $\left[\mathrm{Rb}^{1+}\right]_{8}\left[\mathrm{~B}^{1-}\right]_{8}\left[\mathrm{Si}^{0}\right]_{38}$, respectively. In both cases, the distribution of boron and silicon atoms is exceptional for intermetallic clathrates. Substitution atoms or vacancies in clathrate-I phases usually appear in the tetrakaidecahedral cages first, ${ }^{23}$ but the boron atoms in $\mathrm{K}_{7} \mathrm{~B}_{7} \mathrm{Si}_{39}$ and $\mathrm{Rb}_{8} \mathrm{~B}_{8} \mathrm{Si}_{38}$ solely contribute to the pentagonal dodecahedral cages. In this work, we have prepared the clathrate-I phase $\mathrm{K}_{8-x} \mathrm{~B}_{y} \mathrm{Si}_{46-y}$ with different compositions and studied the crystal structure adaption to the boron content. We show that borosilicide clathrates constitute an own branch of the intermetallic clathrate-I family with a unique atomic order.

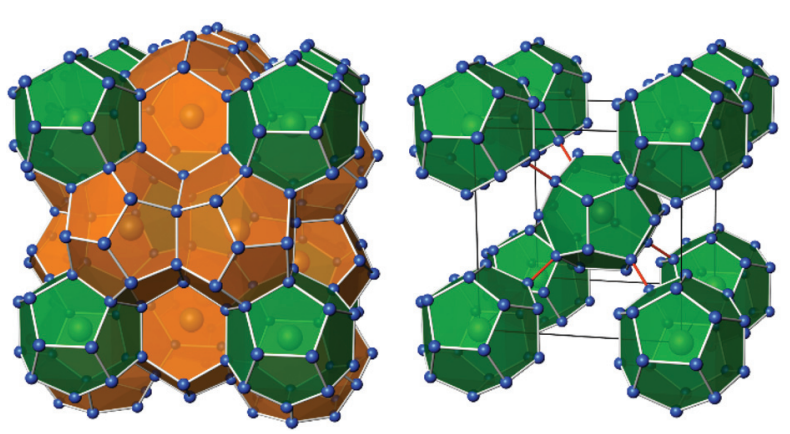

Fig. 1 (Left) Clathrate-I crystal structure of $\mathrm{K}_{8-x} \mathrm{~B}_{y} \mathrm{Si}_{46-y}$. Four-bonded framework atoms form pentagonal dodecahedral cages (green), and tetrakaidekahedral cages (orange). Silicon and boron atoms are drawn blue, potassium atoms in the cages grey. (Right) Partial structure of the dodecahedral cages, connected via B-Si bonds along $\{111\}$ directions (red). 


\section{Results and discussion}

\subsection{Preparation}

A challenge in the preparation of alkali metal borosilicides was the high reactivity of the elemental components with the common crucible materials. Using tantalum ampoules at reaction temperatures of $\approx 1000{ }^{\circ} \mathrm{C}$, annealing times did not exceed 2 days to prevent the formation of byproducts such as $\mathrm{TaB}_{2}$ or $\mathrm{TaSi}_{2}$ (Table 1). For shorter annealing times, the reactivity of boron affected product formation. By using plasma activated amorphous boron, ${ }^{24}$ clathrates can be formed with higher $\mathrm{B}$ and $\mathrm{K}$ content than from precursor alloys of boron and silicon. Although the clathrate product always yielded sharp and symmetric reflections in XRPD, crystallites of the boron-rich samples typically contained inclusions of boron (Fig. 2), which were not removed by washing with aqua regia. Therefore, the boron content of bulk samples was generally higher than the boron content of the clathrate phase. WDX spectroscopy confirmed the ratio $n_{\mathrm{K}} / n_{\mathrm{Si}}$ obtained from crystal structure refinement, and the presence of boron in the clathrate phase (Table 1). The quantification of boron was outside of the reliable measuring range. For the high-pressure product, the WDXS analysis was affected by the small grain size and contamination by the boron nitride crucible material.

Thermogravimetry experiments on a washed clathrate sample revealed an onset of continuous weight loss at $c a$. $870{ }^{\circ} \mathrm{C}$. The sample was not molten a this temperature. The total amount is consistent with the complete elimination of potassium, while the residue showed the reflections of borondoped $\alpha$-Si in XRPD. For comparison, samples were heated in open Ta crucibles enclosed in silica glass ampoules for one day at various temperatures. Under these conditions, the decomposition was observed at similar temperatures, as evidenced by the reaction of potassium vapor with the glass vessel.
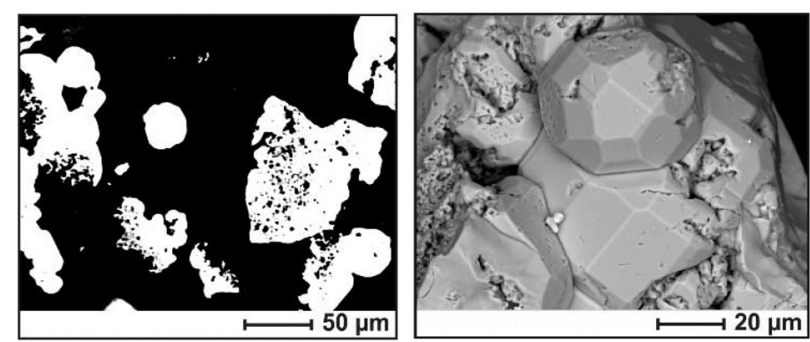

Fig. 2 Microstructure of sample 2 (cf. Table 1); back-scattered electron images. (Left) Polished clathrate grains (white) in a polymer matrix (black); boron inclusions appear as black spots within the white grains. (Right) Surface morphology of the as-grown sample.

\subsection{Crystal structure and composition}

In the first part of this section, we outline the basic structure refinement strategy using crystal 4 (Table 1). The refinement was performed for a binary structure model $\mathrm{K}_{8} \mathrm{Si}_{46-x}$. Assuming the absence of vacancies, the number of Boron atoms was calculated subsequently (see ESI, section $1 \dagger$ ). By refining the boron content directly, the results depended on the refinement strategy. In the starting model, all positions were fully occupied (space group $\operatorname{Pm} \overline{3} n ; 6 \mathrm{Si} 1$ in $6 c, 16 \mathrm{Si} 2$ in $16 i, 24 \mathrm{Si} 3$ in $24 k, 2 \mathrm{~K} 1$ in $2 a, 6 \mathrm{~K} 2$ in $6 d$ ). The refinement with 872 symmetry independent reflections (up to $\sin \theta \lambda^{-1}=1.2$ ) and anisotropic approximation of atomic displacement parameters (ADP) yielded the residual value $R_{\mathrm{F}}=0.085$. Characteristic features of the model at that stage were $U_{\text {eq }}(\mathrm{K} 1)$ $>U_{\text {eq }}(\mathrm{K} 2)$, and $U_{\text {eq }}(\mathrm{Si} 1) \ll U_{\text {eq }}(\mathrm{Si} 3)<U_{\text {eq }}(\mathrm{Si} 2)$. Because the 20 -atom cage of $\mathrm{K} 1$ is smaller than the 24-atom cage of $\mathrm{K} 2$, suggesting the reverse ratio of the ADP, the ratio $U(\mathrm{~K} 1)>U(\mathrm{~K} 2)$ indicates partial site occupancy of K1. Consequently, the site occupancy factor of $\mathrm{K} 1$ was refined to 0.54 , while the $R$-value decreased to $R_{\mathrm{F}}=0.075$, and the displacement parameters converged to the expected ratio $U(\mathrm{~K} 1)<U(\mathrm{~K} 2)$. The ADP values of

Table 1 Lattice parameter a, refined compositions, composition from WDXS and chemical analysis (CA) of the clathrate phase $\mathrm{K}_{8-x} \mathrm{~B}_{y} \mathrm{Si}_{46-y}$ and preparation conditions

\begin{tabular}{|c|c|c|c|c|c|c|c|c|c|}
\hline \multirow[b]{2}{*}{ No. } & \multirow[b]{2}{*}{$a / \AA ̊ \AA$} & \multicolumn{3}{|c|}{ Composition from structure refinement } & \multicolumn{3}{|c|}{ Composition from analysis } & \multicolumn{2}{|l|}{ Preparation } \\
\hline & & Composition & $n(\mathrm{~K}) / n(\mathrm{~B})$ & $n(\mathrm{~K}) / n(\mathrm{Si})$ & & $n(\mathrm{~K}) / n(\mathrm{~B})$ & $n(\mathrm{~K}) / n(\mathrm{Si})$ & $\begin{array}{l}\text { Educts } \\
\text { K: B : Si (at. \%) }\end{array}$ & Conditions $^{a}$ \\
\hline 1 & $9.9366(3)$ & $\mathrm{K}_{7.18(2)} \mathrm{B}_{7.2(5)} \mathrm{Si}_{38.8(5)}$ & $0.9972(20)$ & $0.1851(20)$ & $\begin{array}{l}\text { WDXS } \\
\text { CA }\end{array}$ & $\begin{array}{l}0.79(10) \\
1.048(6)\end{array}$ & $\begin{array}{l}0.181(2) \\
0.1808(12)\end{array}$ & $15.4 / 24.6 / 60.0$ & $\begin{array}{l}900^{\circ} \mathrm{C} \\
48 \mathrm{~h} ; \mathrm{B}_{\mathrm{am}}\end{array}$ \\
\hline 2 & $9.9393(2)$ & $\mathrm{K}_{7.12(4)} \mathrm{B}_{7.1(6)} \mathrm{Si}_{38.9(6)}$ & $1.003(3)$ & $0.183(3)$ & WDXS & $0.91(3)$ & $0.191(4)$ & $20.0 / 40.0 / 40.0$ & $\begin{array}{l}950^{\circ} \mathrm{C} \\
20 \mathrm{~h} ; \mathrm{B}_{\mathrm{am}}\end{array}$ \\
\hline 3 & $9.9470(2)$ & $\mathrm{K}_{6.98(4)} \mathrm{B}_{6.4(5)} \mathrm{Si}_{39.6(5)}$ & $1.108(2)$ & $0.176(2)$ & WDXS & $0.90(3)$ & $0.185(2)$ & $17.9 / 14.3 / 67.9$ & $\begin{array}{l}1000{ }^{\circ} \mathrm{C}^{b} \\
44 \mathrm{~h} ; \mathrm{B}_{\text {cryst }}\end{array}$ \\
\hline 4 & $9.952(1)$ & $\mathrm{K}_{7.08(1)} \mathrm{B}_{6.8(3)} \mathrm{Si}_{39.2(3)}$ & $1.0412(14)$ & $0.1806(14)$ & EDXS & - & $0.1707[1]$ & $20.7 / 13.8 / 65.5$ & $\begin{array}{l}950^{\circ} \mathrm{C} \\
48 \mathrm{~h} ; \mathrm{B}_{\text {cryst }}\end{array}$ \\
\hline 5 & $9.968(1)$ & $\mathrm{K}_{6.80(2)} \mathrm{B}_{6.4(5)} \mathrm{Si}_{39.6(5)}$ & $1.063(2)$ & $0.172(2)$ & $\begin{array}{l}\text { WDXS } \\
\text { CA }\end{array}$ & $\begin{array}{l}0.96(3) \\
1.161(13)\end{array}$ & $\begin{array}{l}0.183(5) \\
0.1687(17)\end{array}$ & $17.3 / 7.7 / 75.0$ & $\begin{array}{l}1000^{\circ} \mathrm{C} \\
56 \mathrm{~h} ; \mathrm{B}_{\mathrm{am}}\end{array}$ \\
\hline 6 & $9.9055(1)$ & $\mathrm{K}_{7.85(2)} \mathrm{B}_{7.8(1)} \mathrm{Si}_{38.2(1)}$ & $1.01(3)$ & $0.204(5)$ & WDXS & $0.815(7)$ & $0.214(2)$ & 41.7/16.7/41.7 & $\begin{array}{l}1000^{\circ} \mathrm{C} \\
5 \mathrm{~h} ; \mathrm{B}_{\mathrm{am}}, 8 \mathrm{GPa}\end{array}$ \\
\hline
\end{tabular}

${ }^{a} \mathrm{~B}_{\mathrm{am}}$ : amorphous boron; $\mathrm{B}_{\text {cryst }}$ crystalline boron. ${ }^{b}$ Preparation in an iron crucible 
K2 indicated a disc-like shape, which adequately reflects the larger volume and the anisotropic shape of the $\mathrm{K} 2$ cage.

Occupancy refinement for the framework positions resulted in a distinctly reduced electron density for $\mathrm{Si} 2$, a minor reduction for $\mathrm{Si} 3$, and a $R_{\mathrm{F}}$ value of 0.042 . The ADP ratio for the framework positions did not change. The results reveal local disorder as the reason for the enhanced ADP of Si2 and $\mathrm{Si} 3$ positions and a mixed occupancy with Si and B atoms. The calculated residual density without occupancy of Si2 displayed a non-spherical distribution (Fig. 3, top left). When the Si2 position was occupied with a with a mixture of Si and B atoms in isotropic approximation, the refinement revealed residual density along the space diagonal (Fig. 3 top right), which was not removed by applying anisotropic ADP (Fig. 3, bottom left). Only by introducing two $16 i$ split positions $(\mathrm{Si}+\mathrm{B})$, the refinement resulted in a 'flat' residual density map and a residual value of $R_{\mathrm{F}}=0.039$. The occupancy of site $\mathrm{Si} 3$ was estimated in a similar fashion. The residual density calculated without atoms at this position revealed a non-spherical distribution in two directions - approximately along the diagonal in the (010) plane and perpendicular to it in (100) (Fig. 4a). Neither anisotropic approximation for ADPs for a single mixed occupied position (Fig. 4b and c) nor a model with two positions located in the (010) plane (Fig. 4d) yielded an acceptable description of the electron density in this region. However, it was satisfactorily described applying two silicon occupied split positions one in the (010) plane $(24 k)$, and another one out of plane (48l) (Fig. 4e). Thereby, the residual value dropped to 0.031 (ESI, Table S4†). The final atomic coordinates and displacement parameters for all crystal structures $\mathrm{K}_{8-x} \mathrm{~B}_{y} \mathrm{Si}_{46-y}$, which were refined applying the same strategy, are listed in the (ESI, Tables S1-S6†).

The preferred substitution of $\mathrm{Si} 2$ by $\mathrm{B}$ atoms at site $16 i$ is a feature that only occurs for clathrate-I borosilicides so far. Typically, the replacement of group 14 elements in intermetallic clathrates by heteroatoms starts at site $6 c$ (Si1). As in all

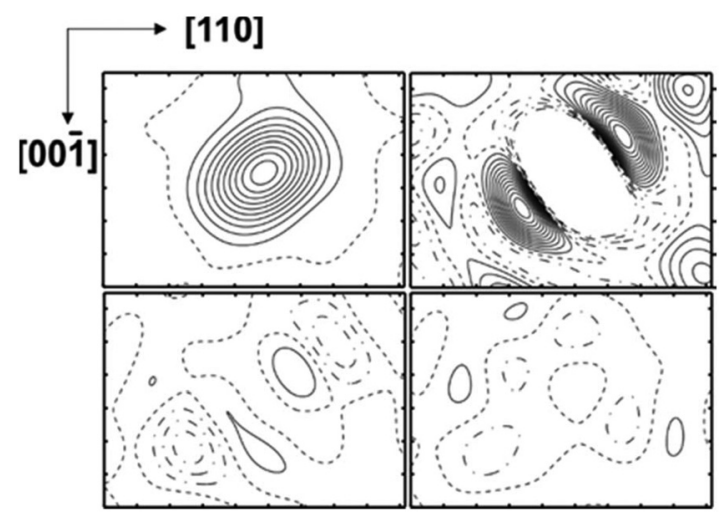

Fig. 3 Distribution of the difference electron density in vicinity of the Si2 position: (top left) without any atoms; (top right) with one atom in isotropic approximation and (bottom left) with one atom in anisotropic approximation; (bottom right) with two mixed occupied positions. The center of each panel is located at $(0.18,0.18,0.18)$. Isolines represent steps of 5 e $\AA^{-1}$ in the top left panel and 0.5 e $\AA^{-1}$ in all other panels.

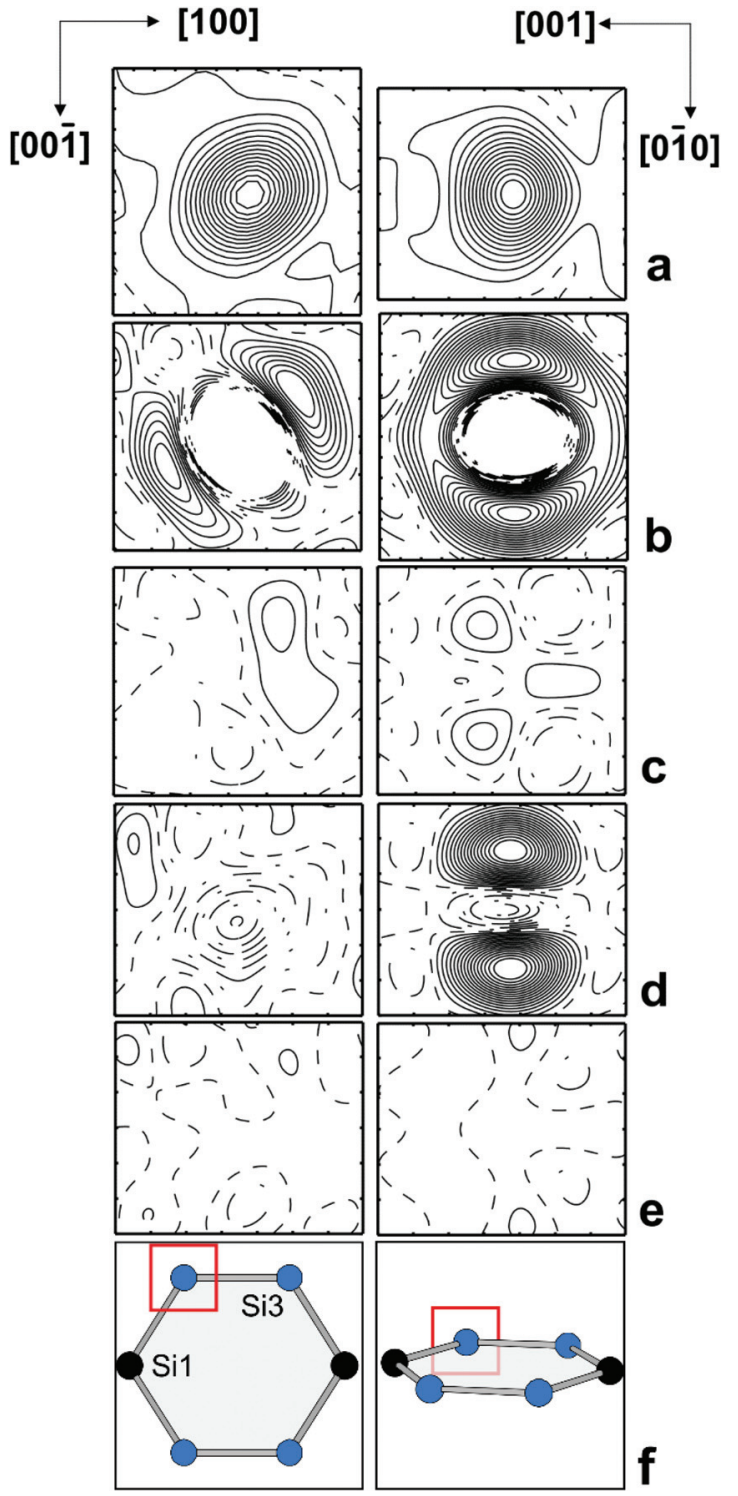

Fig. 4 Distribution of the difference electron density around Si3 in two perpendicular planes: (a) without any atoms; (b) with one mixed occupied position in the (010) plane in isotropic approximation; (c) with one mixed occupied position in the (010) plane in anisotropic approximation; (d) with two mixed occupied positions in the (010) plane in isotropic approximation; (e) with two silicon occupied positions (one on the (010) plane, the other one out of plane, cf. ESI Table 5†); (f) position of the selected planes in the structure. The center of each panel is located at $(0.30,0.00,0.12)$. The step of the isolines is 5 e $\AA^{-3}$ in (a) and 0.5 e $\AA^{-3}$ in all other panels.

binary clathrate silicides $\mathrm{M}_{8-x} \mathrm{Si}_{46}$ the $\mathrm{Si} 2$ atoms show the shortest framework distances, ${ }^{22,23}$ this site should be the most suitable one for boron atoms. However, the partial substitution at site $16 i$ inevitably causes a structural disorder, because $16 i$ atoms appear as bonded pairs. Assuming that direct contacts of negatively charged boron anions are energetically unfavourable, the pairs mainly consist of B-Si contacts in two orientations plus, for $y<8$, a minority of Si-Si pairs. The bond distances $d(\mathrm{~B}-\mathrm{Si}) \approx 2.0 \AA$ and $d(\mathrm{Si}-\mathrm{Si}) \approx 2.3 \AA$ differ substantially. 
The experimental electron density at sites $16 i$ and $24 k$ thus represents a sum of local configurations, which is reflected by the apparently large and anisotropic ADPs (Fig. 3 and 4). After introducing split positions for $\operatorname{Si} 2\left(16 i_{1}+16 i_{2}\right)$ the question arises, which of them is the favourable one for boron atoms (Fig. 5).

When boron occupies the split site $16 i_{1}$, the neighbouring site $16 i_{2}$ is occupied by silicon ( $\mathrm{Si22}$ ). In this case, all B-Si distances are favourably short: one to the silicon atom at $16 i_{2}$, and three to the $\mathrm{Si} 32$ at $48 l$ with values of $\approx 2.0 \AA$. Site $16 i_{2}$ is less favourable for $\mathrm{B}$ atoms because of three longer distances of ca. 2.2 $\AA$ to the Si32 atoms. The minority case without boron atoms is represented by $\mathrm{Si21-Si21}$ pairs. Here, the Si21 atoms are connected to three $\mathrm{Si} 31$ at site $24 k$. The Si22 atoms at $16 i_{2}$ are connected to boron at $16 i_{1}$, to Si31 atoms at $24 k$ or to the $\mathrm{Si} 32$ atoms at $48 \mathrm{l}$ out of the mirror plane. To interpret the refined site occupancies, we extend the split-site model and consider the possible local configurations of the $\mathrm{Si}_{20-x} \mathrm{~B}_{x}$ dodecahedron around K1, which is composed of B21, Si21, Si22, Si31 and Si32 atoms (Fig. 6a-f).

A frequently occurring situation, which cannot be explained in Fig. 5, is that the $\mathrm{Si} 3$ atom has two adjacent $\mathrm{B}$ atoms (Fig. 6a). Here, a shift towards Si32 is no longer possible, because the approach to one of the boron atom would enlarge the distance to the other one. In this case, a short distance to both boron atoms can still be realized by stretching the Si3-Si3 bond and shifting the pair at the same time towards the cage center (Fig. 6b, blue arrows). With equal distances to both boron atoms, such $\mathrm{Si} 3$ atoms contribute to the electron density at Si31 and not, as Fig. 5 may suggest, at Si32. Only in such cases, where the $\mathrm{Si} 3$ atoms are connected to a $\mathrm{B}$ atom and a $\mathrm{Si}$ atom (Fig. 6c and d), they shift towards the B atom and occupy the Si32 position. As a consequence, the Si3-Si3 bond line deviates from the [100] direction. This can even occur for a Si31-Si31 bond when the Si3 atoms are connected to a closer Si21 and a more distant Si22 atom (Fig. 6e and f). In the refined structure model, site Si31 represents all cases, for which the $24 k$ atoms are connected to $16 i$ atoms of the same type (Fig. 6b), while Si32 represents the asymmetrical local

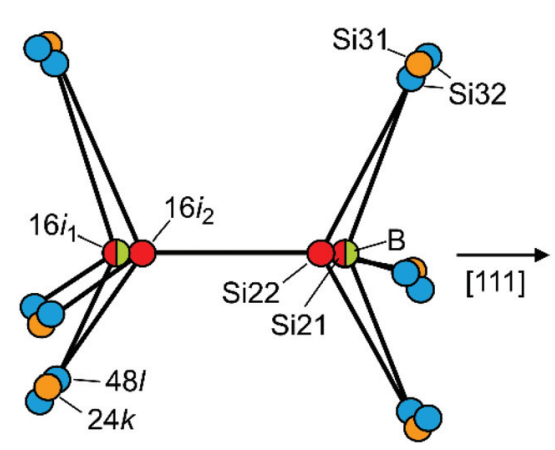

Fig. 5 Local atomic arrangements around the Si2 and Si3 positions with the split sites of $16 i_{1}$ (B and Si21) and $16 i_{2}$ (only Si22). Adjacent Si22 positions are alternatively occupied so that the short distance $d(\mathrm{Si} 22-$ Si22) $=1.8 \AA$ does not occur.

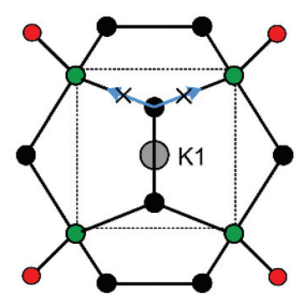

a
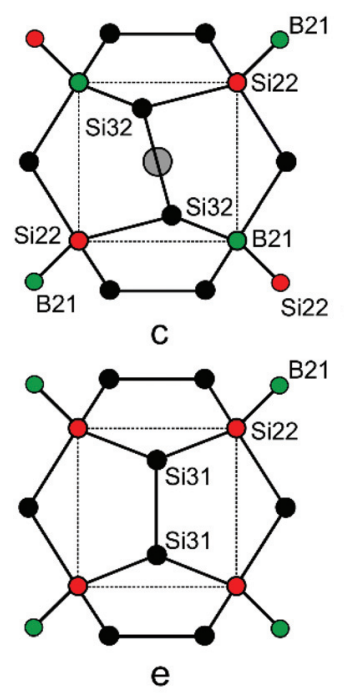

B21

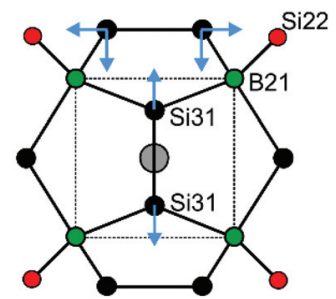

b
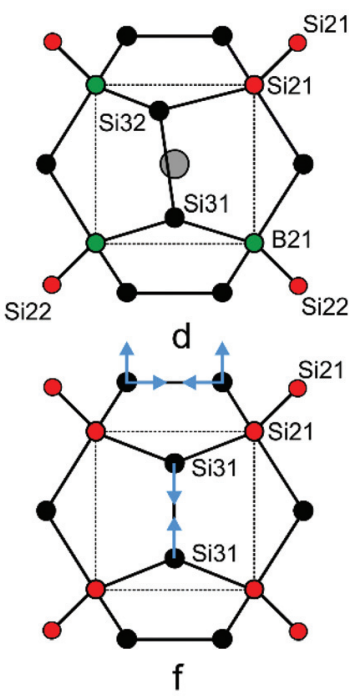

Si31, Si32
Fig. 6 Local atomic arrangements derived from the Si3 split sites in the crystal structure of $\mathrm{K}_{8-x} \mathrm{~B}_{y} \mathrm{Si}_{46-y}$. The examples show how the $\mathrm{Si}_{20-x} \mathrm{~B}_{x}$ dodecahedra may respond to the composition.

configuration (Fig. 6c). As the asymmetric arrangements occur more frequently, the Si32 site (48l) features a higher occupancy than $\operatorname{Si31}(24 k)$.

The crystal structure of the high-pressure product was refined from synchrotron X-ray powder diffraction data. The powder pattern (Fig. 7) was fully indexed with a primitive unit cell so that the structure model was developed in space group $P m \overline{3} n$ applying a similar refinement strategy as described above. The tetrakaidecahedral cages were found fully occupied with $\mathrm{K} 2$, while the $\mathrm{K} 1$ atoms in the smaller dodecahedral showed a minor deficiency. In the framework, position Si1 was fully occupied by silicon, but both $\mathrm{Si} 2$ and also $\mathrm{Si} 3$ showed mixed $\mathrm{B} / \mathrm{Si}$ occupancy. However, the lower resolution of the method did not allow for resolving the split positions. Therefore, the bond distances represent mean values from the superposition of $\mathrm{B}-\mathrm{Si}$ and $\mathrm{Si}-\mathrm{Si}$ bonds. Remarkably, the refined composition $\mathrm{K}_{7.85(2)} \mathrm{B}_{7.8(1)} \mathrm{Si}_{38.2(1)}$ fits the Zintl rule within one experimental error. Thermal analysis of the highpressure product was similar to those of the ambient pressure products and did not show any exothermic decomposition effect on heating, which would have indicated a metastable phase.

The lattice parameter of the borosilicide clathrates with the remarkable minimum for $\mathrm{K}_{7.85(2)} \mathrm{B}_{7.8(1)} \mathrm{Si}_{38.2(1)}(a \approx 9.905 \AA)$ is 


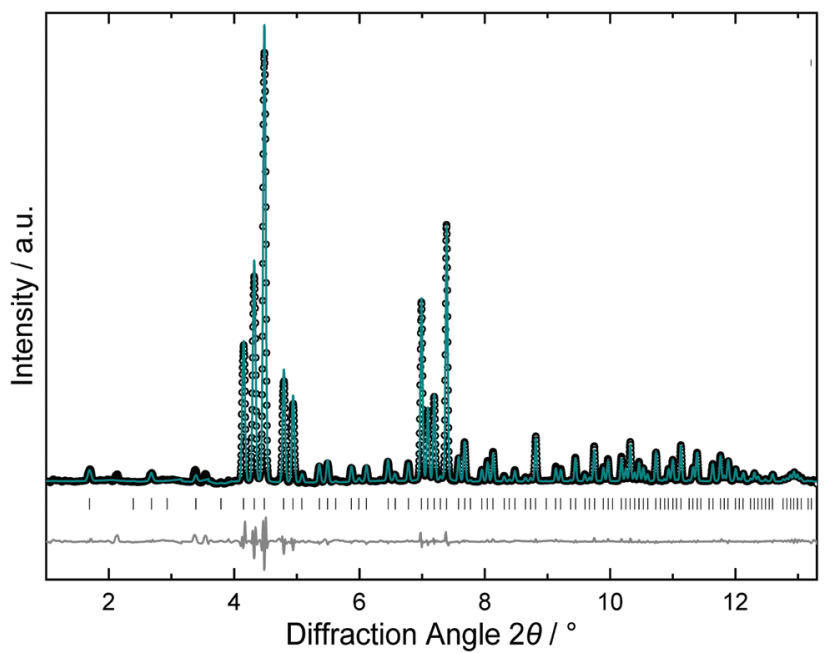

Fig. 7 Powder $X$-ray diffraction pattern of the high-pressure product $\mathrm{K}_{7.85(2)} \mathrm{B}_{7.8(1)} \mathrm{Si}_{38(1)}$ (synchrotron radiation, $\lambda=0.20709 \AA$ A). Black circles represent experimental data, green line the calculated profile, black ticks the reflection positions, the grey line in the bottom the difference profile.

considerably smaller than that of the corresponding binary silicon clathrate $\mathrm{K}_{8-x} \mathrm{Si}_{46}(a \approx 10.26 \AA)$. The lattice parameter decreases with increasing boron content, which is expected from the short $d(\mathrm{~B}-\mathrm{Si})$ distances (Fig. 8). Surprisingly, the decrease is not hindered by the increasing potassium content, which suggests an attractive interaction between the fourbonded boron anions and the K1 cations in the dodecahedral cages. So far, a decrease of lattice parameter with an increasing number of filler atoms has been observed only for the oversized $\mathrm{Si}_{28}$ cages in the clathrate-II structure of $\mathrm{Na}_{24-x} \mathrm{Si}_{136}{ }^{25}$

For compositions with partial occupancy of position K1, the crystal structure comprises empty and filled dodecahedral

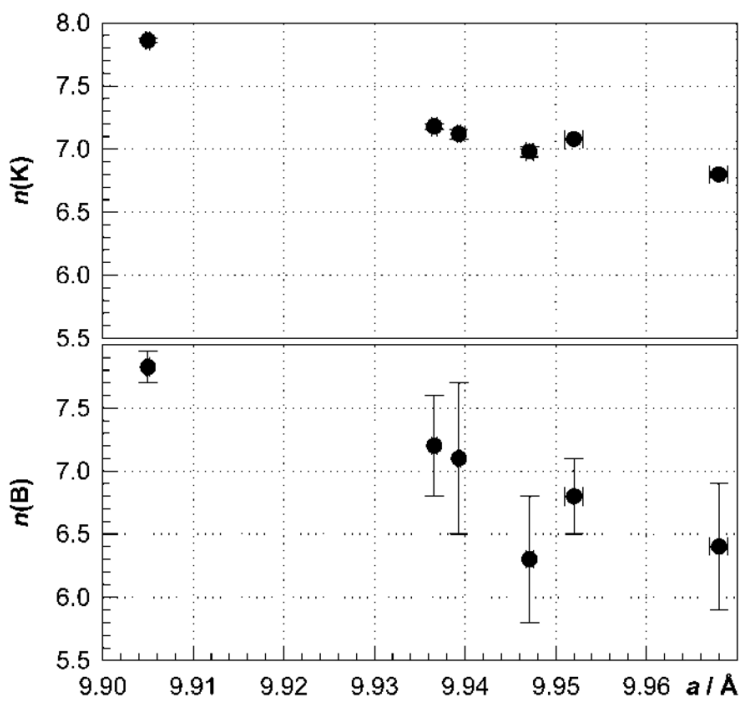

Fig. 8 Number of potassium and boron atoms per unit cell of $\mathrm{K}_{8-x} \mathrm{~B}_{y} \mathrm{Si}_{46-y}$ obtained from crystal structure refinement $v s$. lattice parameter.

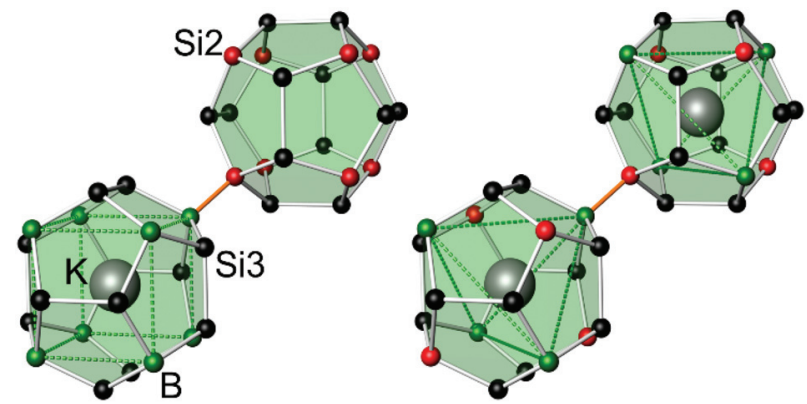

Fig. 9 The dodecahedral cages in $\mathrm{K}_{8-x} \mathrm{~B}_{y} \mathrm{Si}_{46-y}$ are interconnected via $16 i$ atoms along the $\{111\}$ directions (red). (Left) An ideal arrangement for a hypothetical composition $\mathrm{K}_{7} \mathrm{~B}_{8} \mathrm{Si}_{38}$, where $\mathrm{B}$ atoms preferably surround $\mathrm{K} 1$ atoms. (Right) A possible arrangement for completely ordered and fully occupied $\mathrm{K}_{8} \mathrm{~B}_{8} \mathrm{Si}_{38}$.

cages. Hence, an attractive interaction between $\mathrm{K} 1$ and $\mathrm{B}$ atoms would imply that the B atoms of B-Si22 pairs are oriented towards the $\mathrm{K} 1$ atoms. A complete separation into $\mathrm{Si}_{20}$ and $\mathrm{K} 1 @ \mathrm{Si}_{12} \mathrm{~B}_{8}$ cages could be achieved for the hypothetical composition $\mathrm{K}_{7} \mathrm{~B}_{8} \mathrm{Si}_{38}$ (Fig. 9, left). For the nearly filled composition $\mathrm{K}_{7.8} \mathrm{~B}_{7.8} \mathrm{Si}_{38.2}$ a tetrahedral coordination of $\mathrm{K} 1$ by B atoms is most probable (Fig. 9, right). An ordered distribution of boron atoms may cause superstructures. For example, the ideal arrangement of $\mathrm{B}$ atoms derived for the compositions $\mathrm{K}_{7} \mathrm{~B}_{8} \mathrm{Si}_{38}$ corresponds to space group $P m \overline{3}$ (Fig. 9, left), and for $\mathrm{K}_{8} \mathrm{~B}_{8} \mathrm{Si}_{38}$ to space group P4 $\overline{3} n$ (Fig. 9, right). But so far, there is no evidence for a superstructure formation in our experiments. In the local structure, the distortions caused by short distances $d(\mathrm{~B}-\mathrm{Si} 22)$ should favor arrangements with a uniform shortening of all four space diagonals. Moreover, the direct connection of silicon rich dodecahedra (Fig. 6f) is unfavorable due the very short distance Si21-Si31 (ESI, Table S4d†) which might also explain the existence of a lower limit for the boron content in $\mathrm{K}_{8-x} \mathrm{~B}_{y} \mathrm{Si}_{46-y}$.

\subsection{Chemical bonding}

Two ordered models in the space group $P 4 \overline{3} n$ were investigated for the idealized composition $\mathrm{K}_{8} \mathrm{~B}_{8} \mathrm{Si}_{38}$, which is close to the composition $\mathrm{K}_{7.85(2)} \mathrm{B}_{7.8(1)} \mathrm{Si}_{38.2(1)}$ of the high-pressure product. In the acentric space group $P 4 \overline{3} n$, the former position Si2 of space group $P m \overline{3} n$ is represented by two eight-fold positions Si21 and Si22 which are fully occupied either by boron or by silicon atoms (for atomic parameters see ESI, Table S7 $\dagger$ ). In both models, the boron atoms are arranged tetrahedrally around K1 (Fig. 9, right). The positions Si1 and Si3 are occupied by silicon only. For the chosen model, the outer position Si21 is more favorable for boron than Si22 (Fig. 5), which is consistent with the structure discussion above.

In order to study the redistribution of the charges within the framework for different local arrangements, and the charge ratio between boron ad silicon atoms, the analysis of the calculated electron density was performed within the Quantum Theory of Atoms in Molecules. ${ }^{26}$ For both hypothetical models, the calculation reveals that the potassium atoms 


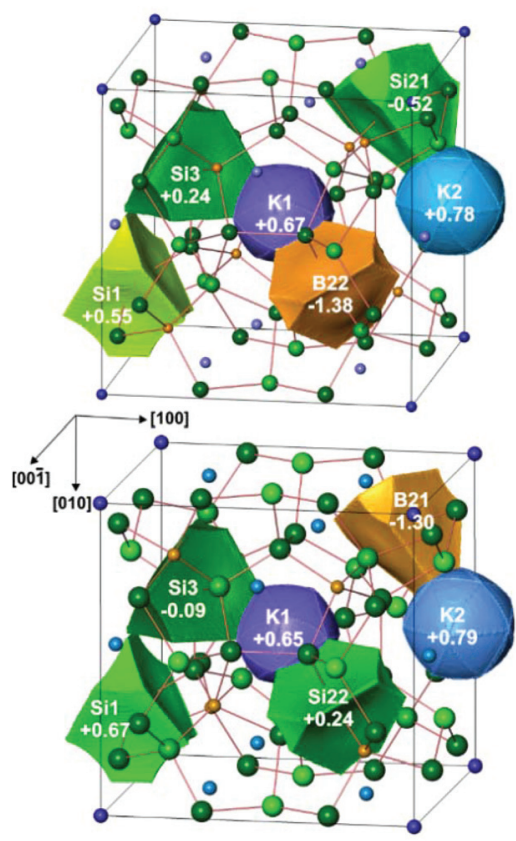

Fig. 10 QTAIM charges in $\mathrm{K}_{8} \mathrm{~B}_{8} \mathrm{Si}_{38}$ models 1 (top) and 2 (bottom) with different distributions of boron and silicon atoms at the $\mathrm{Si} 2$ position.

have nearly the same positive charge and transfer, thus, their valence electron to the framework. Rather unexpectedly, the distribution of the electrons within the framework does not completely follow the Zintl count (Fig. 10). The charges of the four-bonded silicon atoms deviate from the expected zero value and are between -0.52 and +0.55 in model 1 and between -0.09 and +0.67 in model 2 . Independently from the effective silicon charges, boron atoms have more negative charges as expected from the Zintl count and electronegativity.

\section{$2.4{ }^{11} \mathrm{~B}$ NMR and ${ }^{29} \mathrm{Si}$ NMR investigations}

To study the local arrangement of the boron atoms, samples 1 , 3 and 5 (Table 1 ) were investigated by ${ }^{11} \mathrm{~B}$ NMR spectroscopy in static mode. For all samples, one sharp signal with a slightly asymmetric peak shape is observed (Fig. 11, left). The signal
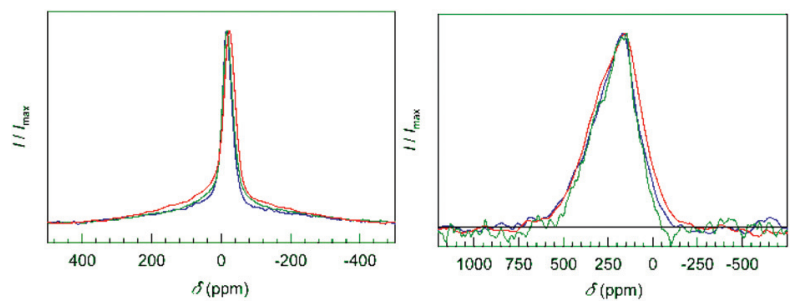

Fig. 11 NMR spectra (static echo measurements) of the clathrates with composition $\mathrm{K}_{7.18(2)} \mathrm{B}_{7.2(5)} \mathrm{Si}_{38.8(5)}$ (blue), $\mathrm{K}_{6.98(4)} \mathrm{B}_{6.4(5)} \mathrm{Si}_{39.6(5)}$ (green) and $\mathrm{K}_{6.80(2)} \mathrm{B}_{6.4(5)} \mathrm{Si}_{39.6(5)}$ (red). (Left) ${ }^{11} \mathrm{~B}$ NMR signals are sharp and slightly asymmetric. The broad background is attributed to the presence of amorphous boron inclusions. (Right) ${ }^{29} \mathrm{Si}$ NMR signals (static echo measurements) show a small Knight shift typical for widely electronically balanced silicon clathrate-I compounds. ${ }^{28-30}$ shape and the shifts of $-15 \mathrm{ppm}$ to $-24 \mathrm{ppm}$ are close to the former result ( -5 ppm for $\mathrm{K}_{7} \mathrm{~B}_{7} \mathrm{Si}_{39}{ }^{21}$ ).

By using echo measurements, contributions of amorphous boron appearing as a broad background signal were separated. For the quantitative analysis of the boron content, the samples were measured at standardized conditions. The integrated intensity of the ${ }^{11} \mathrm{~B}$ NMR signals was normalized by the nominal sample weight as the clathrate content in the bulk samples was always close to $100 \%$. The results confirm the range of boron content found by X-ray diffraction (Table 9, ESI $\dagger$ ). The single, sharp signal points to only one dominating local environment of four-bonded boron atoms. Therefore, the presence of $\mathrm{B}-\mathrm{B}$ bonds besides $\mathrm{B}-\mathrm{Si}$ bonds at site $16 i$ is ruled out. The slight asymmetry in the ${ }^{11} \mathrm{~B}$ signals is explained by the differences in bond angles, bonding distances and local symmetry due to the different connection patterns of the $\mathrm{Si}-\mathrm{B}$ pairs (Fig. 5 and 6). ${ }^{29} \mathrm{Si}$ NMR spectroscopy reveals a broad signal, which does not allow for an assignment of individual contributions of framework sites (Fig. 11, right). The signals have a smaller Knight shift than $\mathrm{Ba}_{8-x} \mathrm{Si}_{46}$ or $\mathrm{Na}_{8} \mathrm{Si}_{46}{ }^{27,31}$ This indicates bad-metal behavior of the clathrate phase for all samples, which is in agreement with electronically widely (but not exactly) balanced chemical compositions. ${ }^{28-30}$

\subsection{Magnetic susceptibility}

The samples 1, 3 and 5 (Table 10, ESI†) show diamagnetic behavior at room temperature, but with an upturn towards low temperatures which is assigned to Curie-like paramagnetic impurities. The extracted temperature independent susceptibility values are negative and similar to those calculated on the basis of diamagnetic increments for $\mathrm{K}^{+}$cations, $\mathrm{B}^{3+}$ and $\alpha-\mathrm{Si}^{32,33}$ for the refined composition. However, the sum of the increments underestimates the diamagnetism, as only the core value is used for boron and the increment of silicon in the anionic framework of the clathrate structure is expected to be more negative than for $\alpha$-Si. Deviation of the measured data toward less negative values (sample 5) are attributed to contributions of conduction electrons (Pauli paramagnetism) caused by $n(\mathrm{~K})>n(\mathrm{~B})$. Sample 3 , prepared in an iron crucible, had a lower purity than the other samples. The result confirms the absence of framework vacancies in the structure as the diamagnetic contributions of $(3 \mathrm{~b}) \mathrm{Si}^{-}$anions ${ }^{23}$ would exceed the elemental increments of $(4 \mathrm{~b}) \mathrm{Si}^{0}$ significantly. ${ }^{34}$

\section{Experimental}

Synthesis. Potassium (Chempur, 99.95\%), silicon (Chempur, 99.9999\%), and crystalline boron (Chempur, 99.95\%) were used without further refinement. Amorphous boron powder (Alfa Aesar), which typically contains oxidic impurities in the range of 1-3 mass\%, was purified in a streaming hydrogen plasma. ${ }^{23}$ For this purpose, the boron powder was placed in a corundum crucible situated in a silica glass Schlenk-tube. Plasma treatment under controlled $\mathrm{H}_{2}$ flow was realized in a microwave furnace (MLS) for 20 minutes $\left(P=1200 \mathrm{~W}, p\left(\mathrm{H}_{2}\right) \approx\right.$ 
2 mbar) at temperatures below $300{ }^{\circ} \mathrm{C}$. The purified boron powder was free of oxygen and hydrogen according to chemical analysis and still amorphous. Preparation of the clathrate phase at ambient pressure proceeded via two different routes. Using amorphous boron, silicon powder was admixed in different atomic ratios. The mixture was homogenized in an agate mortar and directly filled into a tantalum ampoule with an excess of potassium (Table 1). Alternatively, mixtures of crystalline boron and silicon were melted in an arc furnace before grinding. An excess of potassium was placed at the bottom of a tantalum tube and covered by the $\mathrm{B} / \mathrm{Si}$ precursor alloy as described earlier. ${ }^{21}$ In both preparation routes, the sealed tantalum ampoules were placed in quartz glass vessels under Ar atmosphere and heated to $650{ }^{\circ} \mathrm{C}$ for ten hours. After this pre-reaction, the temperature was raised by $20 \mathrm{~K} \mathrm{~h}^{-1}$ to maxima between $900{ }^{\circ} \mathrm{C}$ and $1000{ }^{\circ} \mathrm{C}$. An exception is the sample 3, which was prepared 30 years ago in an iron crucible. The iron content in the sample was ca. $1 \mathrm{wt} \%$ according to WDXS. After annealing for 1-2 days, the ampoules were removed from the hot furnace. While the operations up to this stage were performed either at a Schlenk line or in an argonfilled glove box, the elimination of residual potassium silicide is performed in normal atmosphere with isopropanol in an atmosphere of streaming Ar. After the first, normally violent reaction, the process is continued with ethanol. Residual elemental silicon and boron were removed successively with 1 $\mathrm{M}$ aqueous $\mathrm{NaOH}$ solution and boiling aqua regia, respectively. After washing, the remaining clathrate-I phase showed wellshaped crystals with metallic luster (Fig. 2).

High-pressure preparation started from a finely ground mixture of $\mathrm{K}_{4} \mathrm{Si}_{4}$ and amorphous boron powder with atomic ratio $\mathrm{K}: \mathrm{B}: \mathrm{Si}=5: 2: 5$. The powder was mechanically closed in a boron nitride crucible $(d=3 \mathrm{~mm})$ and plugged in the drilled hole of a $\mathrm{MgO}$ octahedron $(l=18 \mathrm{~mm})$. The synthesis was conducted using a multi-anvil press comprising a Walker-type module. ${ }^{35}$ Before the experiment, pressure and temperature measurements were calibrated by recording resistance changes of bismuth and thermocouple-calibrated runs, respectively. A pressure of at $p=8( \pm 1)$ GPa was applied, and the sample was heated to $T=1273( \pm 127) \mathrm{K}$ within $15 \mathrm{~min}$. After annealing for $60 \mathrm{~min}$, the sample was quenched under load. To remove side products, the sample was first washed with ethanol, then with deionized water and finally with acetone, and dried at room temperature. The high-pressure product is air-stable and inert against strong acids and bases.

Elemental analysis. The clathrate phase is stable against aqua regia and $\mathrm{NaOH}$ solutions. Samples were dissolved in aqueous $\mathrm{HNO}_{3} / \mathrm{HF}$ by using a microwave-assisted procedure (ETHOS, MLS) in closed Teflon vessels and analyzed by using optical emission spectrometry (ICP-OES, VISTA RL, Varian). Standardization was performed with a matrix-matched standard. For determination of $\mathrm{O}$ and $\mathrm{H}$ in amorphous boron, specimens of about $25 \mathrm{mg}$ were loaded into tin capsules and analyzed by carrier gas hot extraction with IR spectrometry of $\mathrm{CO}, \mathrm{CO}_{2}$, and $\mathrm{H}_{2} \mathrm{O}$ (TCH 600, LECO). The carbon content was analyzed after the combustion of the sample in oxygen stream (CHLH 200, LECO).
Powder X-Ray diffraction (PXRD). Diffraction experiments on powder samples were performed with a Guinier camera (Huber G670, $\mathrm{Cu} \mathrm{K} \alpha_{1}$ radiation, $\lambda=1.54056 \AA$, germanium monochromator, $5^{\circ} \leq 2 \theta \leq 100^{\circ}, \Delta 2 \theta=0.005$ ). Reflection positions were corrected by using $\mathrm{LaB}_{6}$ standard (NIST), and the unit cell parameters were calculated from least-square refinement. Rietveld refinements were performed using synchrotron data (Desy Hamburg, PETRA III, Beamline P02.1, $\lambda=$ $0.20720 \AA$ ) recorded at room temperature. All calculations were performed with the WinCSD software. ${ }^{36}$

$\mathrm{X}$-ray single crystal diffraction. Five single crystals obtained from samples with different lattice parameters were investigated by single-crystal X-ray diffraction. The crystals were selected under paraffin oil and fixed onto a glass capillary. The measurements were performed on a IPDS diffractometer (Stoe, MoK $\alpha$ radiation, $\lambda=0.71073 \AA$ ), a CAD4 diffractometer (EnrafNonius, Mo K $\alpha$ radiation) or a Rigaku Spider diffractometer (rotating anode and Varimax optics, AgK $\alpha$ radiation, $\lambda=$ $0.560871 \AA$ ). Absorption correction was performed with a multi-scan procedure. The crystal structures were refined using WinCSD software. ${ }^{36}$ Details concerning the data collection, structure refinement, and interatomic distances are given in the ESI. $\dagger$

Scanning electron microscopy (SEM). Bulk samples were embedded in epoxy resin with conductive carbon filler and polished. Energy-dispersive X-ray spectroscopy (EDXS) was performed with an attached EDAX Si (Li) detector, wavelength dispersive X-ray spectroscopy (WDXS) by using a Cameca SX100 electron microprobe. The $\mathrm{K} \alpha$ lines of the elements were measured and evaluated using $\mathrm{Ni}_{3} \mathrm{~B}, \mathrm{Mg}_{2} \mathrm{Si}$, and $\mathrm{KBr}$ as standards.

Thermogravimetry (TG). The thermal stability of the clathrate phase was investigated using a thermo balance (Netzsch STA 409 CD). The substance was filled in a Ta crucible and heated with a rate of $10 \mathrm{~K} \mathrm{~min}^{-1}$ up to $1200^{\circ} \mathrm{C}$.

Magnetic susceptibility. Specimens of $m=50 \mathrm{mg}-90 \mathrm{mg}$ were measured in closed silica tubes using a SQUID magnetometer (MPMS XL-7, Quantum Design) at external fields of 3.5 $\mathrm{T}$ and $7 \mathrm{~T}$. The diamagnetic contribution of the silica tube was determined prior to the measurements and subtracted. The samples contained tiny amounts of ferromagnetic impurities with a mass content of about 1 ppm, which may originate, e.g., from the usage of steel tools in sample preparation. Therefore, an extrapolation to infinite external field was performed by applying the Honda-Owen method. ${ }^{37}$ Afterward, the temperature-independent contribution was extracted by a polynomial fit.

Nuclear Magnetic Resonance Spectroscopy. NMR experiments were performed with a Bruker Avance 500 spectrometer in a magnetic field of $B_{0}=11.74 \mathrm{~T}$. A standard Bruker Magic Angle Spinning (MAS) probe for $2.5 \mathrm{~mm} \mathrm{ZrO}_{2}$ rotors was used for spinning experiments while the static experiments were performed in probes built by NMR Service GmbH, Erfurt. The external reference frequencies for ${ }^{11} \mathrm{~B}$ and ${ }^{29} \mathrm{Si}$ nuclei were 160.458 MHz and 99.360 MHz, corresponding to the signals of $\mathrm{BF}_{3} \cdot \mathrm{Et}_{2} \mathrm{O}$ and TMS solutions in $\mathrm{CCl}_{3} \mathrm{D}$, respectively. In the case 
of ${ }^{11} \mathrm{~B}$, the signal was collected either after a single pulse or as an echo after two pulses of equal length (6 $\mu \mathrm{s})$ applying a recovery time of $30 \mathrm{~s}-60 \mathrm{~s}$. The single pulse experiments were performed for signal assignment, while the quantitative evaluation bases on the echo measurements. In the case of ${ }^{29} \mathrm{Si}$, Hahn echoes were obtained after a pulse sequence, in which the second pulse had twice the length of the first one $(1.8 \mu \mathrm{s}$ and $3.6 \mu \mathrm{s}$ ), applying a recovery time of $10 \mathrm{~s}$.

Chemical bonding. For the electronic structure calculations, the TB-LMTO-ASA software was used. ${ }^{38}$ Analysis of the chemical bonding is performed by the electron localizability approach in position space. ${ }^{39}$ The electron localizability indicator (ELI) in its ELI-D representation ${ }^{40}$ is calculated together with the electron density (ED), using a specialized module implemented in the TB-LMTO-ASA package. The topology of the three-dimensional distributions of ELI-D and ED was evaluated with the program DGrid. ${ }^{41}$ Integration of the electron density in the basins bounded by zero-flux surfaces of the electron density or ELI-D gradient fields yield the atomic charges or bond populations, respectively. This procedure follows the quantum theory of atoms in molecules (QTAIM). ${ }^{26}$

\section{Conclusions}

Different compositions of the clathrate borosilicide $\mathrm{K}_{8-x} \mathrm{~B}_{y} \mathrm{Si}_{46-y}$ were investigated by combining X-ray diffraction experiments, solid state NMR and magnetic susceptibility measurements. Borosilicide clathrates constitute an own branch of intermetallic clathrates with the unique structural feature that boron atoms first substitute atoms at site $16 i$ and not at site $6 c$ as it is observed in other clathrate-I phases. The complex structural order was investigated and a structure model was established. The correlation between lattice parameter, boron and potassium content points to an attractive interaction between potassium cations and four-bonded boron anions. The unit cell volume shrinks distinctly with boron content. In particular, the sample with the highest boron content, $\mathrm{K}_{7.8} \mathrm{~B}_{7.8} \mathrm{Si}_{38.2}$, features an extraordinary small lattice parameter compared to other clathrate-I silicides ( $a=9.9055(1)$ $\AA)$. By trend, the compositions within the homogeneity range follow the Zintl-rule with $n(\mathrm{~K}) \approx n(\mathrm{~B})$. Chemical bonding analysis with the electron localizability indicator reveals the Zintllike behavior of the $\mathrm{B}^{-}$anions.

\section{Conflicts of interest}

There are no conflicts to declare.

\section{Acknowledgements}

The authors would like to thank the competence groups structure and metallography at MPI CPfS for providing experimental support. Open Access funding provided by the Max Planck Society.

\section{References}

1 The Physics and Chemistry of Inorganic Clathrates, ed. G. S. Nolas, Springer Series in Materials Science, 2014.

2 J. Dolyniuk, B. Owens-Baird, J. Wang, J. V. Zaikina and K. Kovnir, Mater. Sci. Eng., R, 2016, 108, 1.

3 S. Cros and M. Pouchard, C. R. Chim., 2009, 12, 1014.

4 M. Christensen, S. Johnsen and B. B. Iversen, Dalton Trans., 2010, 39, 978.

5 B. Eisenmann, H. Schäfer and R. Zagler, J. Less-Common Met., 1986, 118, 43.

6 C. L. Condron, J. Martin, G. S. Nolas, P. M. B. Piccoli, A. J. Schultz and S. M. Kauzlarich, Inorg. Chem., 2006, 45, 9381.

7 C. L. Condron, S. M. Kauzlarich, T. Ikeda, G. J. Snyder, F. Haarmann and P. Jeglic, Inorg. Chem., 2008, 47, 8204.

8 E. N. Nenghabi and C. W. Myles, J. Phys.: Condens. Matter, 2008, 20, 415214.

9 J. H. Roudebush, C. de la Cruz, B. C. Chakoumakos and S. M. Kauzlarich, Inorg. Chem., 2012, 51, 1805.

10 M. Bobnar, B. Böhme, M. Wedel, U. Burkhardt, A. Ormeci, Y. Prots, C. Drathen, Y. Liang, H. D. Nguyen, M. Baitinger and Y. Grin, Dalton Trans., 2015, 44, 12680.

11 M. Troppenz, S. Rigamonti and C. Draxl, Chem. Mater., 2017, 29, 2414.

12 J. Martin, H. Wang and G. S. Nolas, Appl. Phys. Lett., 2008, 92, 222110.

13 E. S. Toberer, M. Christensen, B. B. Iversen and G. J. Snyder, Phys. Rev. B: Condens. Matter Mater. Phys., 2008, 77, 075203.

14 G. S. Nolas, J. L. Cohn, G. A. Slack and S. B. Schujman, Appl. Phys. Lett., 1998, 73, 178.

15 B. C. Chakoumakos, B. C. Sales, D. G. Mandrus and G. S. Nolas, J. Alloys Compd., 2000, 296, 80.

16 B. C. Sales, B. C. Chakoumakos, R. Jin, J. R. Thompson and D. G. Mandrus, Phys. Rev. B: Condens. Matter Mater. Phys., 2001, 63, 245113.

17 S. Paschen, W. Carrillo-Cabrera, A. Bentien, V. H. Tran, M. Baenitz, Yu. Grin and F. Steglich, Phys. Rev. B: Condens. Matter Mater. Phys., 2001, 64, 214404.

18 A. Czybulka, B. Kuhl and H.-U. Schuster, Z. Anorg. Allg. Chem., 1991, 594, 23.

19 Ya. Mudryk, P. Rogl, C. Paul, S. Berger, E. Bauer, G. Hilscher, C. Godart and H. Noël, J. Phys.: Condens. Matter, 2002, 14, 7991.

20 A. Bentien, E. Nishibori, S. Paschen and B. B. Iversen, Phys. Rev. B: Condens. Matter Mater. Phys., 2005, 71, 144107.

21 W. Jung, J. Loerincz, R. Ramlau, H. Borrmann, Yu. Prots, F. Haarmann, W. Schnelle, U. Burkhardt, M. Baitinger and Yu. Grin, Angew. Chem., Int. Ed., 2007, 46, 6725, (Angew. Chem., 2007, 119, 6846).

22 J. M. Hübner, W. Jung, M. Schmidt, M. Bobnar, P. Koželj, B. Böhme, M. Baitinger, M. Etter, Yu. Grin and U. Schwarz, Inorg. Chem., 2020, DOI: 10.1021/acs.inorgchem.0c02357.

23 M. Baitinger, B. Böhme, F. R. Wagner and U. Schwarz, Z. Anorg. Allg. Chem., 2020, 646, 1034.

24 A. Alekseeva, K. Kovnir, P. Chizhov, M. Baitinger and Yu. Grin, European Patent, EP1893320B8, 2010. 
25 M. Beekman, E. N. Nenghabi, K. Biswas, C. W. Myles, M. Baitinger, Yu. Grin and G. S. Nolas, Inorg. Chem., 2010, 49, 5338.

26 F. W. Bader, Atoms in Molecules: A Quantum Theory, Oxford University Press, New York, 1990.

27 R. Castillo, W. Schnelle, M. Bobnar, U. Burkhardt, B. Böhme, M. Baitinger, U. Schwarz and Y. Grin, Z. Anorg. Allg. Chem., 2015, 641, 206.

28 H. Sakamoto, H. Tou, H. Ishii, Y. Maniwa, E. A. Reny and S. Yamanaka, Physica C, 2000, 341, 2135.

29 Y. Maniwa, H. Sakamoto, H. Tou, Y. Aoki, H. Sato, F. Shimizu, H. Kawaji and S. Yamanaka, Mol. Cryst. Liq. Cryst., 2000, 341, 497.

30 B. Böhme, M. Bobnar, A. Ormeci, K. Peters, W. Schnelle, M. Baitinger and Yu. Grin, Z. Kristallogr. - Cryst. Mater., 2017, 232, 223.

31 J. He, D. D. Klug, K. Uehara, K. F. Preston, C. I. Ratcliffe and J. S. Tse, J. Phys. Chem. B, 2001, 105, 3475.
32 P. W. Selwood, Magnetochemistry, Interscience, New York, USA, 2nd edn, 1956, p. 78.

33 S. Hudgens, M. Kastner and H. Fritzsche, Phys. Rev. Lett., 1974, 33, 1552.

34 Y. Liang, B. Böhme, A. Ormeci, H. Borrmann, O. Pecher, F. Haarmann, W. Schnelle, M. Baitinger and Yu. Grin, Chem. - Eur. J., 2012, 18, 9818.

35 D. Walker, M. A. Carpenter and C. M. Hitch, Am. Mineral., 1990, 75, 1020.

36 L. Akselrud and Yu. Grin, J. Appl. Crystallogr., 2014, 47, 803.

37 K. Honda, Ann. Phys., 1910, 337, 1027.

38 O. Jepsen, A. Burkhardt and O. K. Andersen, The Program TB-LMTO-ASA. Vers. 4.7, Stuttgart, 1999.

39 M. Kohout, Int. J. Quantum Chem., 2004, 97, 651.

40 F. R. Wagner, V. Bezugly, M. Kohout and Yu. Grin, Chem. Eur. J., 2007, 13, 5724.

41 M. Kohout, DGrid, versions 4.6-5.0, 2018. 